\title{
Taxonomy, truth-value gaps and incommensurability: a reconstruction of Kuhn's taxonomic interpretation of incommensurability
}

\author{
Xinli Wang \\ Philosophy Department, Juniata College, Huntingdon, PA 16652, USA
}

Received 8 January 2001; received in revised form 5 June 2001

\begin{abstract}
Kuhn's alleged taxonomic interpretation of incommensurability is grounded on an ill defined notion of untranslatability and is hence radically incomplete. To supplement it, I reconstruct Kuhn's taxonomic interpretation on the basis of a logical-semantic theory of taxonomy, a semantic theory of truth-value, and a truth-value conditional theory of cross-language communication. According to the reconstruction, two scientific languages are incommensurable when core sentences of one language, which have truth values when considered within its own context, lack truth values when considered within the context of the other due to the unmatchable taxonomic structures underlying them. So constructed, Kuhn's mature interpretation of incommensurability does not depend upon the notion of truth-preserving (un)translatability, but rather depends on the notion of truth-value-status-preserving crosslanguage communication. The reconstruction makes Kuhn's notion of incommensurability a well grounded, tenable and integrated notion.
\end{abstract}

(C) 2002 Elsevier Science Ltd. All rights reserved.

Keywords: Incommensurability; Thomas Kuhn; Taxonomic structures; Lexicons; Truth-value; Untranslatability; Cross-language communication

\section{Incommensurability as communication breakdown}

Thomas Kuhn observed that a reader often encounters apparently meaningless passages when he or she attempts to understand some old scientific texts. ${ }^{1}$ These

E-mail address: wang@juniata.edu (X. Wang).

${ }^{1}$ Kuhn (1983), p. 669; Kuhn (1987), pp. 8-12; Kuhn (1988), pp. 9-11; Kuhn (1991), p. 4. 
apparently nonsensical passages cannot be simply taken as evidence of the author's confused expressions or mistaken beliefs, nor as evidence of the interpreter's personal limitation of knowledge or lack of skills of interpretation. It is a communication breakdown between two scientific communities as a whole, rather than between two individuals with different dialects, intentions or even conflicting interests. It seems to involve some deep semantic and conceptual obstruction between two competing scientific languages. ${ }^{2}$ Deeply perplexed by these observations, Kuhn set out to give a philosophical explication of such communication breakdowns that he dubbed 'incommensurability'.

According to a naïve but widely accepted assumption, any effective communication $^{3}$ between two language communities requires some appropriate common measure between the languages used. However, controversy arises as to what counts as an appropriate common measure necessary for effective communication between two language communities. Kuhn spent some thirty-five years trying to pin it down. Although Kuhn's position underwent dramatic changes, there was a thread through all the changes. He sought to identify a significant necessary common measure of effective communication, and perhaps primarily to deny that such an identified common measure exists, thereby identifying a certain kind of semantic obstruction between would-be communicants.

Consider the development of Kuhn's position since the publication of his Structure of scientific revolutions in 1962 . Kuhn initially specified the common measure as a shared paradigm: the entire constellation of shared metaphysical commitments, shared problems to solve, shared methodological standards of adequacy, and shared perceptions. Faced with extensive criticism, Kuhn realized later that his concept of paradigm was too vague, and so he concentrated on an essential part of the paradigm, namely exemplars and similarity relationships among items determined by exemplars. ${ }^{4} \mathrm{He}$ accordingly specified the common measure as shared similarity relationships (family resemblance) among objects or situations for categorization. ${ }^{5}$ However, the major problem with the identification of similarity relationships as a common measure between two competing languages is that a mere shift of similarity relationships does not necessarily cause a communication breakdown.

\footnotetext{
${ }^{2}$ A comprehensive scientific theory can be roughly regarded as a well developed conceptual system. The linguistic system employed to express it is what is usually called a scientific language. In fact, the language of a scientific theory can be considered as an interpreted formal language by identifying the truths of the language under interpretation with the theorems of the theory. In addition, a scientific theory can be coded in different natural languages; and two different scientific theories can be coded in the same natural language. Incommensurability obtains between two scientific theories or their languages, but not between two natural languages.

${ }^{3}$ Communication breakdown does not amount to the failure of understanding. Mutual understanding is necessary, but not sufficient, for effective communication. The speaker of a language may understand another incommensurable language, in the manner of a bilingual, but fail to communicate with the other side effectively.

4 Kuhn's early position is represented in Kuhn (1970a,b, 1976, 1977b, 1979).

5 Kuhn (1970a), pp. 200-201; Kuhn (1970b), pp. 275-276; Kuhn (1976), p. 195; Kuhn (1979), p. 416; Kuhn (1987), pp. 20-21.
} 
To further specify the structure of categorical frameworks of scientific languages and to explore their role in effective communication between two competing language communities has constituted the central task of Kuhn's explication of incommensurability ever since. After 1983, "the phrase "no common measure" became "no common language". The claim that two theories are incommensurable is then the claim that there is no language, neutral or otherwise, into which both theories, conceived as sets of sentences, can be translated without residue or loss' (Kuhn, 1983, p. 670). After 1987, Kuhn gave up the common language requirement as too broad, and focused exclusively on one essential part of a language-its taxonomic structure. ${ }^{6}$ At this latest stage, the phrase 'common measure' became 'shared lexical structure or taxonomy ${ }^{7}$ between two competing scientific languages (Kuhn, 1991, p. 4; Kuhn, 1993, p. 326).

It is clear that Kuhn's notions of incommensurability evolved in the process of his effort to specify a significant common measure of effective cross-language communication. Cross-language communication breakdown is the essential sense of Kuhn's notion of incommensurability. ${ }^{8}$ To say that two scientific theories are incommensurable is, for Kuhn, to say that a necessary common measure is lacking between the languages employed by them and that thereby the effective cross-language communication between their opponents breaks down. Left unresolved however is (a) the specification of such a significant common measure necessary for effective communication and (b) the explication of why such a specified common measure is indeed necessary for effective cross-language communication. Presumably we need some sort of theory of cross-language communication to resolve these matters. Without it any explication of incommensurability is radically incomplete.

\section{The accepted reading of Kuhn's taxonomic interpretation}

An understanding of Kuhn's apparent resolution can be gleaned through the accepted reading of his latest position, that is, the taxonomic interpretation of incom-

\footnotetext{
${ }^{6}$ Kuhn (1983), p. 683; Kuhn (1988), pp. 9, 16; Kuhn (1991), pp. 4-5, 9; Kuhn (1993), pp. 323-326.

7 A taxonomic structure/lexical structure (briefly, a lexicon) is the conceptual/vocabulary structure shared by all members of a language community that provides the community with both shared taxonomic categories/kind-terms and shared similarity/dissimilarity relationships between them. For a detailed explication of Kuhn's concepts of taxonomy, taxonomic structures and kind-terms, see Hacking (1993).

8 There are two kinds of incommensurability. On the one hand, a radical conceptual shift in a scientific language within the same cultural or intellectual tradition can make it hardly intelligible to a later age Especially when two successive rival scientific theories are separated by a scientific revolution, their advocates are liable to experience a communication breakdown. On the other hand, substantial semantic and/or conceptual disparities between two comprehensive theories embedded in two coexistent, distinct, intellectual/cultural traditions can create serious impediments to communication. It might be argued that Kuhn is particularly interested in succession of ideas in the history of some particular science-such as the phlogiston theory versus oxygen theory of combustion. However, the second kind of incommensurability (such as Chinese medical theory versus Western medical theory) is arguably at least as important as the first kind, and both are subject to the same interpretation. It will be more productive to show how Kuhn's taxonomic interpretation could apply to the second kind of incommensurability also.
} 
mensurability. As early as the end of the1960s, Kuhn started to think that 'untranslatability' is a better word than 'incommensurability' for what he had in mind when he spoke of a communication breakdown between the users of two alien texts. ${ }^{9}$ After 1983 , by virtue of introducing a new semantic tool of taxonomic structures, Kuhn continued to rely heavily on the notion of untranslatability in his explication of incommensurability. ${ }^{10}$ Translatability became, for Kuhn, a bridge connecting shared taxonomic structures with full cross-language communication. This reading of Kuhn may be concisely formulated as follows: ${ }^{11}$

1. Effective communication between the speakers of two competing scientific languages is possible only if translation between the languages can be carried out. ${ }^{12}$

2. Translation between two scientific languages is possible only if there is a systematic referent-mapping of the corresponding kind-terms in the two languages.

3. A systematic referent-mapping of the corresponding kind-terms between two scientific languages is possible only if the two languages share the same taxonomic structure. ${ }^{13}$

4. Therefore, a shared taxonomic structure is necessary for effective communication between two scientific language communities. ${ }^{14}$

This reading of Kuhn's taxonomic interpretation is one version of what is usually referred to as the translation-failure interpretation of incommensurability. It posits that incommensurability reduces to untranslatability arising from a radical variance of meaning/reference of the terms in two competing scientific languages. Elsewhere it has been argued that this untranslatability concept of incommensurability is flawed (Wang, 1998). Very briefly put, the argument is as follows. (a) There is no tenable and integrated notion of (un)translatability that can be used to clarify the notion of incommensurability. This is because the notion of truth-preserving (un)translatability itself does not have any independent interpretative power and is a philosophically uninteresting notion in the discussion of incommensurability. (b) More significantly, translation is neither sufficient nor necessary for effective cross-language communication. Therefore, reference to untranslatability neither identifies nor resolves the problem of incommensurability, but rather leads to confusion and misunderstanding. It follows from this reasoning that Kuhn seems to face a grave difficulty. On the one hand, without appeal to the notion of untranslatability, Kuhn's taxonomic interpretation is radically incomplete due to a conceptual gap between taxonomic

\footnotetext{
9 For Kuhn's early version of the thesis of untranslatability, see Kuhn (1970a,b, 1976, 1979).

${ }^{10}$ Kuhn (1983), pp. 669-670; Kuhn (1988), p. 11; Kuhn (1991), p. 5.

${ }^{11}$ More precisely, it is not just one accepted reading, but rather one face of Kuhn. Kuhn is notorious for his terminological confusions and constant changing of position on the topic of incommensurability. Even in this latest stage, he still swung between two different interpretations, namely, the translationfailure interpretation and the new interpretation to be presented below.

12 Kuhn (1977a), p. 338; Kuhn (1983), p. 683; Kuhn (1988); Kuhn (1991), p. 5.

${ }^{13}$ Kuhn (1983), p. 683; Kuhn (1988), p. 22; Kuhn (1991), p. 5; Kuhn (1993), p. 324.

${ }^{14}$ Kuhn (1988), p. 16; Kuhn (1991), pp. 4-5; Kuhn (1993), pp. 325-326.
} 
structures of languages and cross-language communication. On the other hand, untranslatability cannot be used to fill the gap.

To escape from this dilemma, it would seem necessary to reconstruct a central line of argument formed in the most recent writings of Kuhn, with an eye to rendering a different reading of Kuhn's taxonomic interpretation. ${ }^{15}$ Although one may find many reconstructions or reinterpretations of Kuhn's concept of incommensurability in the literature, ${ }^{16}$ none has paid much attention to the interpretation developed below, which is arguably more coherent, tenable and powerful. It will be shown that Kuhn has developed implicitly a sort of truth-value-conditional theory of communication, which provides a badly needed cognitive connection between taxonomic structures of languages and cross-language communication. So construed, Kuhn's concept of incommensurability is seen to depend not upon the notion of untranslatability, but on a set of semantic conceptions such as taxonomic structures, truthvalue status and truth-value gaps, possible worlds, and cross-language communication.

\section{Truth-value status and the emergence of truth-value gaps}

Based on his notion of style of reasoning, Ian Hacking suggests that we switch our attention from the notion of truth to the notion of truth-or-falsity in the discussion of incommensurability and related issues (Hacking, 1982, p. 49). Following Hacking, Kuhn admits the importance of the distinction between the notion of truth-value and the notion of truth-value status (whether or not a sentence has a truth-value) in his latest interpretation of incommensurability.

Since that time, I have been gradually realizing (the reformulation is still in process) that some of my central points are far better made without speaking of statements as themselves being true or as being false. Instead, the evaluation of a putatively scientific statement should be conceived as comprising two seldomseparated parts. First, determine the status of the statement: is it a candidate for true/false? To that question, as you'll shortly see, the answer is lexicon-dependent. And second, supposing a positive answer to the first, is the statement rationally assertable? To that question, given a lexicon, the answer is properly found by something like the normal rules of evidence. (Kuhn, 1991, p. 9)

Here Kuhn switches from the classical bivalent semantics to a trivalent semantics

\footnotetext{
15 Perhaps the interpretation to be developed below cannot be found explicitly in Kuhn's writings since it was not fully worked out by the late Kuhn. It is a reconstruction that uses various hints from his latest available writings, which reveal Kuhn's genuine insight into the concept of incommensurability.

16 Such as Balzer (1989), Biagioli (1990), Brown (1983), Chen (1997), Doppelt (1978), Fu (1995), Hoyningen-Huene (1990), Hung (1987), Malone (1993), Ramberg (1989) and Sankey (1991), to mention only a few.
} 
in which a declarative sentence is true, false, or neither-true-nor-false. ${ }^{17}$ As will be demonstrated, it is the notion of truth-value status, instead of truth-preserving translation, that plays the most essential role in Kuhn's new taxonomic interpretation of incommensurability.

In a trivalent semantics, it is possible for a sentence to be neither true nor false, which corresponds to a lack of a classical truth-value (truth or falsity). In this case, it can be said that there is a truth-value gap regarding the sentence within a bivalent semantics. If a substantial number of core sentences ${ }^{18}$ of one scientific language, when considered within the context of a competing language, lack classical truthvalues, then there is a truth-value gap between the two languages. This is what has really happened during scientific revolutions.

Each lexicon makes possible a corresponding form of life within which the truth or falsity of propositions may be both claimed and rationally justified, ... With the Aristotelian lexicon in place, it does make sense to speak of the truth or falsity of Aristotelian assertions in which terms like 'force' or 'void' play an essential role, but the truth values arrived at need have no bearing on the truth or falsity of apparently similar assertions made within the Newtonian lexicon. (Kuhn, 1993, pp. 330-331)

When a Newtonian finds Aristotelian sentences hard to understand, the trouble is not that she/he thinks Aristotle wrote falsely, but that she/he cannot attach truth or falsity to a great many of the Aristotelian core sentences because the Aristotelian lexicon presupposed by the sentences fails when considered within the Newtonian language. Consequently a truth-value gap occurs between the Newtonian language and the Aristotelian language.

Such occurrences of truth-value gaps abound in the history of science. 'Though the originals were candidates for true/false, the historian's later restatements-made by a bilingual speaking the language of one culture to the members of another-are not' (Kuhn, 1991, p. 9). Consider the following two Newtonian sentences about simultaneity and precedence (Gaifman, 1984).

(A) Event $\mathrm{e}_{1}$ and event $\mathrm{e}_{2}$ are simultaneous: $\tau\left(\mathrm{e}_{1}\right)=\tau\left(\mathrm{e}_{2}\right)$.

(B) Event $\mathrm{e}_{1}$ precedes event $\mathrm{e}_{2}: \tau\left(\mathrm{e}_{1}\right)<\tau\left(\mathrm{e}_{2}\right)$.

$A$ and $B$ make perfect sense and are true-or-false in Newtonian physics since, for Newton, physical events happen within a self-existing, ordered line of time points independent of any event. However, according to the relativity theory, precedence

\footnotetext{
17 For a trivalent semantics, see Wang (1999a).

${ }^{18}$ For Kuhn, core sentences of a scientific language are the sentences presupposing the lexicon of the language, such as 'Element $a$ contains more phlogiston than element $b$ ' in the phlogiston theory, 'All diseases are due to the loss of the balance between the yin part and the yang part of the human body' in Chinese medical theory, 'A body without external forces on it tends to seek its natural place' in Aristotelian physics, 'Planets revolve about the earth' in the Ptolemaic astronomy, and so on.
} 
may depend on the coordinate system from which the events are viewed. More precisely, if in some coordinate system the events are separated by distance $d$ and time $\Delta \mathrm{t}$ and $\mathrm{d}>\mathrm{c} \times \Delta \mathrm{t}(\mathrm{c}=$ light velocity $)$, then their temporal order depends on the coordinate system. Therefore, to ask 'Does event $\mathrm{e}_{1}$ precede $\mathrm{e}_{2}$ ?' or 'Are $\mathrm{e}_{1}$ and $\mathrm{e}_{2}$ simultaneous?' without specifying a coordinate system is to ask a factually meaningless question. This is because the notion of absolute simultaneity presupposes the existence of an absolute time ordering which is denied by the relativity theory. Thus $A$ and $B$ have no truth-values from the relativistic point of view. Therefore, the disagreement on the truth-value status of some substantial sentences such as $A$ and $B$ represents the real difference between Newton's and Einstein's concepts of time.

It is useful at this point to move from consideration of cases of two successive rival scientific theories within the same intellectual tradition (as illustrated above) to a case of two comprehensive theories embedded in coexistent, distinct intellectual/cultural traditions. Imagine that a Chinese physician diagnoses a patient's painful spleen as due to an excess of the yin within his spleen (an asthenic spleen) by claiming that An excess of the yin within a person's spleen causes a painful spleen,

on the basis of a fundamental principle of Chinese medical theory, namely, all diseases are due to the loss of the balance between the yin part and the yang part of the human body.

What is the likely response of a practitioner of Western medicine? She/he would certainly not claim that the Chinese assertion is false. The content of $C$ and $D$ lies outside the Westerner's conceptual reach because she/he could not appreciate the way in which the assertion was proposed and justified. It is not even clear to her/him whether the sentence really asserts anything. It is, hence, very likely that she/he would say something like 'What is the point of what the Chinese is saying?'. The Westerner's response implies that the issue of whether the assertion was true or false simply did not arise.

Similar analysis can be extended to the other core sentences of Chinese medical theory. There is no way to match what the Chinese physician wants to say against anything the Western physician wants to say at the theoretical level. They do not lie in the sphere of disagreement or conflict of the sort arising when one theory holds something to be true that the other holds to be false. The difference between them is not that Western medical theory has a different theory of the operation of the yin and the yang from that of its Chinese counterpart or that Chinese physicians say different things about bacteria and viruses, but that one side has nothing to say about them. It is not that they say the same thing differently, but rather they say totally different things. The key contrast here is between saying something (asserting or denying) and saying nothing. The Western physician can neither assert nor deny what is claimed by the Chinese physician. Consequently, the Western physician does 
not regard as false many core sentences of Chinese medical theory; she/he simply cannot assign truth-values to them. ${ }^{19}$

\section{Truth-value status and taxonomic structures}

It is necessary to explain the occurrence, if any, of a truth-value gap between two competing scientific languages. ${ }^{20}$ Kuhn's attempt relies on presenting a sort of theory of truth. The usual theories of truth, such as the correspondence theory, are semantic theories about truth conditions and can only be used to determine the truth-value of a statement. But at issue here is not whether a statement is true but rather whether a given string of words is assertable (hence qualifies as a statement) or whether a sentence has a truth-value. What is needed is not a theory about truth conditions but a theory about truth-value conditions (whether a sentence has a truth-value). A usual theory of truth does not help us with this. So as to distinguish such a theory of truth from the usual theories of truth, it is useful to dub the former a 'theory of truthvalue'. Kuhn did not work out a complete theory of truth-value, but rather provided some clues here and there. Based on these clues, the task remains to reconstruct a Kuhnian theory of truth-value.

\subsection{Taxonomic structures and possible worlds ${ }^{21}$}

Following the conventional possible-world semantics, Kuhn regards a possible world as a way our actual world might have been (Kuhn, 1988, p. 13). The problem is what concept of world is in play here. As P. Hoyningen-Huene argues persuasively, Kuhn uses the concept of world in more than one sense (Hoyningen-Huene, 1993, Ch. 2). It could refer to a world that is 'already perceptually and conceptually subdivided in a certain way' (Kuhn, 1970a, p. 129), which may be called a 'world-for-us'. A world-for-us has a certain conceptual structure imposed by a certain taxonomic structure. It is such a world to which we actually have conceptual access. In another sense, the concept of world could refer to the world-in-itself, namely, what is left over after we subtract all perceptual and conceptual structures imposed by human contributions from a world-for-us. Different from Kant's thing-in-itself, Kuhn stipulates the world-in-itself to be spatiotemporal, not undifferentiated, and in some sense causally efficacious. Beyond this nothing can be said about this world.

In what sense does Kuhn use the concept of a possible world? Do possible worlds include worlds-for-us only or both worlds-for-us and the world-in-itself? Answering

\footnotetext{
${ }^{19}$ For a detailed case study on traditional Chinese medical theory versus contemporary Western medical theory, see Wang (1999b), Sect. 3.1.

${ }^{20}$ It is a long-standing controversial issue as to how truth-value gaps can be admitted in general. Readers can refer to Williamson (1994) and Wang (1999b), Ch. 4, for discussion as to whether truth-value gaps are both stable and well motivated.

${ }^{21}$ Kuhn (1970b), pp. 268, 270-271, 274; Kuhn (1983), p. 683; Kuhn (1987), pp. 20-21; Kuhn (1988), pp. 11, 13-14, 22-24; Kuhn (1991), pp. 5, 10, 12; Kuhn (1993), pp. 319, 330-331.
} 
this question requires consideration of a controversial issue on the ontological status of possible worlds. What is the proper range of possible worlds over which quantification occurs? David Lewis quantifies over the entire range of worlds that have been or might be conceived. Saul Kripke, at the other extreme, quantifies over only the worlds that can be stipulated. Kuhn stands along with Kripke and contends that only a world that can be conceptually accessible in the sense that it can be stipulatable in some language can be a possible world (Kuhn, 1988, p. 14). Since the world-initself is totally inaccessible conceptually to any language community, it is not a possible world in Kuhn's sense. Accordingly, the actual world in which one scientific community lives and works is a possible world that they actually perceive to be.

Notice that in Kuhn's concept of possible worlds, the alleged distinction between there being a possible world and it being conceptually accessible is blurred. For a world to be a possible world it has to be conceptually accessible somehow by some language. Some lexical structures are prerequisite to the existence of, not just accessible to, any possible world. 'Like Kantian categories, the lexicon supplies preconditions of possible experience' (Kuhn, 1991, p. 12). To echo G. Berkeley's famous expression ('to be is to be perceived'), Kuhn would say that to be a possible world is to be conceptually accessible.

Nevertheless, this does not mean that a possible world is conceptually accessible to any language. To be a possible world is to be a world accessible to certain languages, but not to any language. This is because, according to Kuhn, there is a conceptual connection between the taxonomic structure of a certain language and its conceptual accessibility relation to certain possible worlds. First of all, Kuhn contends that acquisition of a certain taxonomic structure is prerequisite to gaining conceptual access to a certain possible world. Certain learned similarity-dissimilarity relationships, as the central feature of a certain taxonomic structure, constitute a languageconditioned way of perceiving a certain world. Until we have acquired them, we cannot perceive that world at all. Similarly, some set of kind-terms supplies necessary categories to describe a certain possible world. For example, in order to gain conceptual access to the Newtonian world, the taxonomic structure of Newtonian mechanics, especially the interrelated kind-terms such as 'force', 'mass' and 'weight', must be possessed first.

Kuhn further specifies that only the possible worlds stipulatable in, or describable by, the lexicon of a language are conceptually accessible to the language community. ${ }^{22}$ This is because 'only the possible worlds stipulatable in that language can be relevant to them. Extending quantification to include worlds accessible only by resort to other languages seems at best functionless, and in some applications it may be a source of error and confusion ... the power and utility of possible-world argu-

\footnotetext{
${ }^{22}$ Kuhn makes a general distinction between conceivable (possible) worlds and stipulatable (possible) worlds. On the one hand, not all the worlds stipulatable within a given lexicon are conceivable. A world containing square circles can be stipulated but not conceived. On the other hand, not all possible worlds conceivable by the speaker of a language are stipulatable in it. For instance, the possible worlds described by the Newtonian language are not stipulatable, although conceivable, in the language of relativity theory See Kuhn (1988), pp. $14 f$.
} 
ments appears to require their restriction to the worlds accessible with a given lexicon, the world that can be stipulated by participants in a given language-community or culture' (Kuhn, 1988, p. 14).

The question arises: 'Why must every aspect of a conceptually accessible possible world be stipulatable in a language? Some of the possible worlds we are interested in might have an unaccessed or inaccessible feature for which no vocabulary has been, or even could be, developed. But it is conceivable. Presumably the actual world is like this'. Obviously this objection assumes that any conceivable world would be conceptually accessible. ${ }^{23}$ But if it is possible to conceive of there being possibilities that cannot be conceived of, then to equate conceivability with conceptual accessibility may make it impossible to deal with such cases. More to the point, the matter at issue here is a language's, not an individual interpreter's, conceptual accessibility relation to a possible world. Whether a possible world is stipulatable in a language can be determined by its taxonomic structure. But conceivability usually refers to a mental state of an individual interpreter. Any possible world would be conceivable for an interpreter if she/he wills to learn and adopt other languages that provide conceptual access to that world. Therefore, the concept of conceivability cannot help us to clarify a language's conceptual accessibility relation to a possible world. It is irrelevant for the purpose at hand.

Theoretically, a language's taxonomic structure enables the community to gain conceptual access to many, even infinitely many, possible worlds that are stipulatable in it. Of course, of these conceptually accessible possible worlds, only a small fraction are evidently possible for the community, which can be confirmed with experiments and observations accepted by the community. Discovering evidently possible worlds is what each scientific community undertakes to do in the course of normal science. As time passes, more and more conceptually possible worlds are excluded by requirements of internal consistency or of conformity with empirical data. Eventually, each lexicon may identify a highly limited set of possible worlds - the possible worlds that are both stipulatable and verifiable within the lexicon-and eventually a single world that the language community conceives as the actual world.

One and the same possible world may be conceptually accessible using different, but compatible lexicons. Languages with incompatible taxonomic structures have access to different possible worlds. 'To possess a lexicon, a structured vocabulary, is to have access to the varied set of worlds which that lexicon can be used to describe. Different lexicons-those of different cultures or different historical periods, for example_-give access to different sets of possible worlds, largely but never entirely overlapping' (Kuhn, 1988, p. 11).

To sum up, according to Kuhn, conceptual accessibility to possible worlds is taxonomic-structure-dependent. Only the possible worlds stipulatable in a language can be conceptually accessible to the language community. And only a world concep-

\footnotetext{
${ }^{23}$ Conceivability is at most, and should be, a necessary condition of conceptual accessibility. A world that cannot be conceived of, such as a world containing square circles, is not a conceptually accessible possible world.
} 
tually accessible to a language is a possible world for it. Since conceptual accessibility to a world is language-dependent, a possible world is language-dependent. Therefore, a world may be possible for some language, but not possible for another. By providing the language community with a network of possibilities, it is the taxonomic structure of a language that determines what is genuinely possible for the language. For this reason, "what I have been calling a lexical taxonomy might, that is, better be called a conceptual scheme, where the "very notion" of a conceptual scheme is not that of a set of beliefs but of a particular operating mode of a mental module prerequisite to having beliefs, a mode that at once supplies and bounds the set of beliefs it is possible to conceive' (Kuhn, 1991, p. 5).

\subsection{Truth-values and possible worlds}

Kuhn contends, following Putnam and many others, that although the correspondence theory of truth (that is, the idea that the substantial nature of truth consists in correspondence with the mind-independent world) has to be given up, the intuition behind it (that is, that the truth of a sentence is determined by its correspondence to a state of affairs external to the sentence) seems too obvious to be put to rest. Such an innocuous intuition can still remain at the heart of a theory of truth as long as 'a world-for-us' is substituted for 'the mind-independent world' at one side of the correspondence relationship (Kuhn, 1988, p. 24; Kuhn, 1991, pp. 6, 8). 'If, as standard forms of realism suppose, a statement's being true or false depends simply on whether or not it corresponds to the real world-independent of time, language, and culture-then the world itself must be somehow lexicon-dependent' (Kuhn, 1988, p. 24).

More precisely, following Wittgenstein's concept of fact-ontology that the world is the totality of facts, ${ }^{24}$ we could treat a Kuhnian possible world as a set of internally related possible facts. Since a Kuhnian possible world is taxonomic-structure-dependent, the possible facts are taxonomic-structure-dependent. It is possible that some state of affairs counts as a possible fact in one language, but not in another with a sufficiently different taxonomic structure. Accordingly, a fact can be defined as the actualization of a possible fact or a possible fact verified in the actual world perceived by a language community. If a fact is the actualization of a possible fact and a possible fact is language-dependent, then a fact seems to be inevitably languagedependent. A fact so defined is relative to a language and subsists in a world specified by the language. There are no mind-independent facts out there waiting to be discovered. $^{25}$

According to the fact-based interpretation of the correspondence theory, a statement is true if and only if it corresponds to a fact. Because whether a state of affairs counts as a fact is dependent upon the taxonomic structure of a language, the same

\footnotetext{
${ }^{24}$ For a good defense of Wittgenstein's fact-ontology as opposite to Aristotelian thing-ontology, see Gaifman (1975, 1976).

${ }^{25}$ For an extensive argument for the relativity of facts, see Wang (1999b), Sect. 9.3.
} 
state of affairs may count as a fact in one language but not in another. Consequently, evaluation of the truth-value of a statement or a truth claim of a sentence is a correlate of taxonomic structure (Kuhn, 1988, p. 24; Kuhn, 1991, p. 4). ${ }^{26}$

\subsection{Truth-value status and possible worlds}

It is time now to answer the question why some sentences which have truth-values in one scientific language lose their truth-values in another. ${ }^{27}$ As pointed out earlier, the truth claim of a sentence $P$ in a language $\mathrm{L}$ with a taxonomic structure TS consists in correspondence to a fact in the actual world perceived by TS. More precisely, take the designate of sentences as 'states of affairs'. The truth-value of $P$ consists in whether the state of affairs designated by $P$ corresponds to a fact admitted by $L$. A fact admitted by $L$ is at least a possible fact from the viewpoint of $L$. Then, in order for $P$ to have a truth-value in $\mathrm{L}$, the state of affairs designated by $P$ has to correspond to a possible fact in a possible world specified by TS of L. For Kuhn, a possible fact from the viewpoint of $\mathrm{L}$ has to be conceptually accessible by TS. Thus the truth-value-status of $P$, when considered within $\mathrm{L}$, is determined by whether the state of affairs designated by $P$ corresponds to a possible fact from the point of view of L. If it does, then $P$ is a candidate for truth-or-falsity; if it does not, then $P$ is not a candidate for truth-or-falsity. Furthermore, if the state of affairs designated by $P$ is not only a possible fact but also a fact specified by $\mathrm{L}$, then $P$ is true. Otherwise it is false.

This establishes that the truth-value status of sentences is internalized within taxonomic structures of a scientific language and becomes taxonomic-structure-dependent. This is why Kuhn claims that 'each lexicon makes possible a corresponding form of life within which the truth or falsity of propositions may be both claimed and rationally justified' (Kuhn, 1993, p. 330). 'Where the lexicons of the parties to discourse differ, a given string of words will sometimes make different statements for each. A statement may be a candidate for truth/falsity with one lexicon without having that status in the others. And even when it does, the two statements will not be the same: though identically phrased, strong evidence for one need not be evidence for the other' (Kuhn, 1991, p. 9). For instance, to assert sentence $D$ is to presuppose that there exist two natural forces 'yin' and 'yang'. This state of affairs designated by $D$ corresponds to a possible fact conceptually accessible by the Chinese medical lexicon. $D$ thus has a truth-value, no matter whether it is actually true or false, from the viewpoint of Chinese medical theory. However, the apparently same sentence is

\footnotetext{
${ }^{26}$ To claim that the evaluation of the truth-value of a statement or a truth claim is lexicon-dependent does not mean that truth itself is relative to language. The core of Kuhn's taxonomic relativist view can be fully preserved by the claim that incompatible taxonomic structures may yield different truth claims. Readers may profit from a similar interpretation, in Hacker (1996), of conceptual relativism based on the distinction between truth itself and truth claims.

${ }^{27}$ Kuhn does not address this matter explicitly. Nevertheless, based on his semantics of possible worlds and his adoption of a modified correspondence notion of truth as presented above, it is conceivable that Kuhn would accept the following solution.
} 
not conceptually accessible by the Western medical lexicon, therefore it does not correspond to any possible fact from the viewpoint of Western medical theory. So $D$ is not simply false, but rather has no truth-value.

\section{The role of truth-or-falsity in cross-language communication}

It has been argued that the truth-value status of sentences is, for Kuhn, taxonomicstructure-dependent based on his theory of truth-value. Kuhn needs to further explore the role of truth-value status in cross-language communication in order to explain why, how and in what sense a shared taxonomic structure between two scientific languages is necessary for effective communication between them.

The lexicons of the various members of a speech community may vary in the expectations they induce, but they must all have the same structure. If they do not, then mutual incomprehension and an ultimate breakdown of communication will result ... If, on the other hand, cores or lexical structure differ, then what appears to be disagreement about fact proves to be incomprehension. The wouldbe communicants have encountered incommensurability, and communication breaks down in an especially frustrating way. (Kuhn, 1993, p. 326)

To begin with, according to Davidson's truth-conditional theory of understanding, to grasp the factual meaning (not just meaning in general) of a sentence is to know its truth conditions. To know the truth conditions of a sentence presupposes that the sentence has a truth-value. That means that having a truth-value is prerequisite for a sentence to be factually meaningful. If a sentence lacks a truth-value when considered within a language, then it will become factually meaningless to its speaker (Davidson, 1984). This explains why Kuhn has observed that 'a historian reading an out-of-date scientific text characteristically encounters passages that make no sense' (Kuhn, 1988, p. 9). This is because these out-of-date sentences, which must have been either true or false in the original text, may be impossible to be stated as candidates for truth-or-falsity at all from today's perspective.

Kuhn provides a different kind of argument for the necessity of truth-or-falsity in cross-language communication (Kuhn, 1991, pp. 8-10). Presumably, in order to make an informative use of a language, the language community has to adhere to some minimal rules of logic. Among them, the law of non-contradiction is crucial. The law claims that $(P \& \sim P)$ is logically false for any sentence $P$ in a language $\mathrm{L}$, or in symbols, $\vdash_{\mathrm{L}} \sim(P \& \sim P)$. The essential function of the law is to forbid accepting both a sentence and its contrary. Thus obedience to the law of non-contradiction is a minimal requirement for any successful linguistic communication or any productive language game.

However, the proper function of the law of non-contradiction can be fulfilled only under some restrictions. When a sentence $P$ has a truth-value within one language $\mathrm{L}_{1}, \vdash_{\mathrm{L}_{1}} \sim(P \& \sim P)$ is valid. But if $P$ is neither true nor false within another language $\mathrm{L}_{2},(P \& \sim P)$ is not false, but rather neither true nor false. The formula 
$\vdash_{\mathrm{L}_{2}} \sim(P \& \sim P)$ thus is invalid since $\sim(P \& \sim P)$ is untrue (neither true nor false) within $\mathrm{L}_{2}$. This shows that the law applies only to the sentences with truth-values. It requires that the sentences involved in discourse must have truth-values or be factually meaningful for one side to effectively communicate with the other. In this sense, to accept sentences in discourse as candidates for truth-or-falsity (which still allows for disagreement about their truth-values) constitutes the minimal rule of any successful linguistic communication. To declare a sentence in discourse as a candidate for truth-or-falsity is to declare a commitment to the law of non-contradiction and to participate effectively in linguistic communication. On the contrary, the law prohibits the occurrence of truth-valueless sentences in normal discourse. To deny the sentences in discourse as candidates for truth-or-falsity is to violate the law of non-contradiction, and thereby to put communication at risk. If a person breaks the rule by denying that the sentences in discourse have truth-values, then she/he declares her/himself outside the language community. If a group of members of a language community deny that the core sentences of the language have truth-values but still try to continue to claim a place in the community, then the communication between them and the rest of the community breaks down. Neither side engages in successful communication even if they seem to talk to one another.

More significantly, it follows that if the core sentences in one scientific language, which are true or false in the language, have no truth-value when considered within another competing language, then there is a truth-value gap between them. The occurrence of such a truth-value gap indicates that effective communication between the two language communities breaks down in a particularly frustrating way. A fully factually meaningful sentence within one language community sounds so strange in the other that it is not factually meaningful and thereby cannot be effectively understood in the latter. Therefore, the occurrence of a truth-value gap between two languages can be used as a strong linguistic correlate of a communication breakdown between them.

Based on the above consideration, Kuhn contends that all language games are no less than true-or-false games. For Kuhn, who endorses Horwich's minimal theory of truth (Horwich, 1990), the truth predicates 'is true' or 'is false' exist primarily for the sake of such a logical need, or to assure that we stick to a language game. In Kuhn's own words:

On this view [a version of the redundancy theory of truth-author], as I wish to employ it, the essential function of the concept of truth is to require choice between acceptance and rejection of a statement or a theory in the face of evidence shared by all ... In this reformulation, to declare a statement a candidate for true/false is to accept it as a counter in a language game whose rules forbid asserting both a statement and its contrary ... In one form or another, the rules of the true/false game are thus universals for all human communities. (Kuhn, 1991, p. 9)

The idea that a language game is a truth-or-falsity game can be viewed as one plausible interpretation of what Wittgenstein was driving at with his metaphor of concep- 
tual schemes as 'language games'. It is truth-value conditions, instead of truth conditions, that are 'the rules of a language game' in this interpretation. This presents a striking contrast to conventional interpretations that focus on truth conditions, such as Davidson's truth-conditional theory of understanding.

A careful reader might have noticed that many sentences under discussion, such as sentences $A, B, C$ and $D$, have been apparently treated throughout the paper unequivocally as well formed sentences across two competing languages. It has been taken for granted that, although semantics (such as truth-value status) is relative to a specific language, syntax is more universal across different languages. But it is doubtful, one might argue, whether there exists a set of rules of syntax that transcends a specific language and determines grammaticality by identifying what counts as a well formed sentence independent of considerations of semantics of language. There is a more radical view, put forward by Wittgenstein and others, that grammar is also internal to a language. On this view, sentence $C$ or $D$ might well be regarded, when considered within the language of Western medical theory, as not a well formed sentence at all, rather than a well formed sentence that lacks a truth-value.

This is not a place to get involved in such a long-debated issue in depth. But I have to admit the significance of such a concern since it seems to point to another possible way of construing Kuhn's taxonomic interpretation of incommensurability. ${ }^{28}$ It needs to be pointed out here that this alternative reading of Kuhn would bring us directly back to the accepted reading of Kuhn's taxonomic interpretation, that is, to one version of the translation-failure interpretation of incommensurability. According to this reading, if syntax is language-dependent, as semantics is, then well formed sentences in one language are likely to be ill formed when considered within a rival language. If so, two rival languages will be mutually untranslatable. ${ }^{29}$

\section{Unmatchable taxonomic structures}

It becomes clear now why shared or matchable taxonomic structures between two scientific languages are necessary for effective communication between them. But

\footnotetext{
28 An anonymous referee brought this to my attention.

${ }^{29}$ Nevertheless, this reading would turn out to be more provocative if it actually meant to identify a different source of truth-value gaps between two competing languages. On the one hand, declarative sentences could be truth-valueless due to the structure of the sentences, such as ill formed sentences (for example, 'sat Kanrog subbppp on') or sentences containing unspecified parameters (for example, 'It is a heap'). Core sentences of one language could lose their truth-values if they are, when considered within another competing language, regarded as ill formed. On the other hand, sentences could lose truth-values due to the failure of their semantic presuppositions. For example, a sentence of the phlogiston theory, 'Element $a$ contains more phlogiston than element $b$ ', is truth-valueless when considered within the oxygen theory since its semantic presuppositions, such as its lexicon including the kind-term 'phlogiston', fail. In this case we have a well formed, truth-valueless cross-language sentence. According to Kuhn, not all truth-value gaps lead to cross-language communication breakdowns, but only the truth-value gaps due to the failure of semantic presuppositions do (a lexicon being one kind of semantic presuppositions).
} 
how can we know whether two taxonomic structures are matchable or not? The following passages from Kuhn provide a hint for such a distinction:

What members of a language community share is homology of lexical structure. Their criteria need not be the same, for those they can learn from each other as needed. But their taxonomic structures must match, for where structure is different, the world is different, language is private, and communication ceases until one party acquires the language of the other. (Kuhn, 1983, p. 683)

Incommensurability thus becomes a sort of untranslatability, ${ }^{30}$ localized to one or another area in which two lexical taxonomies differ. The differences which produce it are not any old differences, but ones that violate either the no-overlap condition, the kind-label condition, or else a restriction on hierarchical relations that I cannot spell out here. (Kuhn, 1991, p. 5; my emphasis)

Full appreciation of these passages necessitates recollection of the two crucial features of Kuhn's kind-terms, namely, the projectibility principle and the no-overlap principle. According to the former, each kind-term is clothed with expectations about its extension or referents. The expectations about a kind-term's referents are projectible in the sense that they enable members of the language community to postulate/project the use of the term to other unexamined situations, including counterfactual situations. ${ }^{31}$ The no-overlap principle says that no two kind-terms at the same level of a (stable) taxonomic tree may overlap in their extensions.

The no-overlap principle is, in fact, the natural result of the projectibility principle. The expectations about a kind-term's (say, 'planets') referents (planets) are usually learned in use. ${ }^{32}$ Presented with exemplars (for example, the sun) drawn from various examined situations, the members of a language community (for example, the Ptolemaic community) acquire a learned expectation about the similarity relationships between the objects or situations that populate the world perceived by them. In terms of these expectations about the similarity relationship among tokens, the members of the community can tell which presentations belong to which kind and which do not (for example, Mars belongs to the kind of planets but the earth does not in the Ptolemaic community). Since people can acquire the same kind-term in different ways, the expectations about the referents of the same kind-term may differ from individual to individual in a language community. However, within the same language community, these different expectations are compatible in the sense that they would eventually identify the same extension for the term by effectively learning

\footnotetext{
${ }^{30}$ Although Kuhn continued to use the term 'untranslatability' in his explication of incommensurability after 1987, he used the term in a different sense from the traditional notion of truth-preserving translation. It is the notion of truth-value-preserving translation that the late Kuhn had in mind when he connected untranslatability with incommensurability.

31 Strictly speaking, to say that a kind-term is projectible is to say that the expectation about a kindterm's referents, rather than a kind-term itself, is projectible.

${ }^{32}$ Kuhn (1987), pp. 20-21; Kuhn (1988), pp. 14-23; Kuhn (1993), pp. 317-318, 325-326.
} 
each other's expectations. On the other hand, some expectations about the referents of a kind-term may be so different in two competing language communities that they are incompatible with one another such that the members from one community (say, the Aristotelian community) will occasionally apply a kind-term (say, 'motion') to a token (say, the growth of an oak) to which the other (say, the Newtonian) community categorically denies that it applies. Usually, the non-identical extensions of the kind-term with incompatible expectations will overlap partially (say, for the movement of a physical object). If the speakers of the two communities use the term 'motion' separately in their own domain, no problem arises. But if they try to engage in an on-going dialogue, the difficulty arises in the region where both apply. Calling the same token 'motion' in the overlap region will always induce two conflicting expectations. Since these expectations are projectible, they cannot be only restricted within the overlap region and will be naturally extended to the respective non-overlap regions (say, to the growth of an oak). Therefore, ultimately the overlap is unstable and eventually only one kind-term remains within one language community (Kuhn, 1993, p. 318).

The reason why two kind-terms at the same level of a (stable) taxonomic tree cannot overlap can be seen more clearly with some high-level theoretical kind terms ${ }^{33}$ clothed with two incompatible expectations. Because these kind-terms figure importantly in fundamental laws about nature, they bring with them nomic expectations - that is, exceptionless generalizations. In science, where they mainly function, these generalizations are usually laws of nature, such as Boyle's law for gases or Newton's laws of motion (Kuhn, 1993, pp. 316-317). Then, if the extensions of such a kind-term with different concepts (for example, 'planets' in Ptolemaic astronomy and in Copernican astronomy) overlap somehow and a token (such as, 'the earth') lies in the overlap region, it would be subject to two exceptionless incompatible natural laws (i.e. the Ptolemaic and Copernican laws of motion of celestial bodies). Similarly, the kind-term 'mass' in either Newton's or Einstein's language brings with it a nomic expectation in the form of a law of nature. Since the laws of nature built into the concept of mass in the Newtonian language and the Einsteinian language are incompatible, the respective expectations associated with the term are incompatible. These incompatible expectations will result in difficulties in the region where Newtonian 'mass' ( mass $_{n}$ ) and Einsteinian 'mass' (mass ${ }_{e}$ ) both apply. Calling some stuff in the overlap region ' mass $_{\mathrm{n}}$ ' induces the nomic expectation associated with either the law of gravity or the second law of motion, while calling the same stuff 'mass, ${ }_{\mathrm{e}}$ 'induces the incompatible nomic expectation associated with the new natural law in Einsteinian theory (general relativity theory). Hence such an overlap cannot remain stable over time (Kuhn, 1988, pp. 14-23). For this reason, "periods in which a speech community does deploy overlapping kind-terms end in one of two ways: either one entirely displaces the other, or the community divides into two,

\footnotetext{
${ }^{33}$ Kuhn uses 'high-level theoretical kind-terms' to refer to those kind-terms (such as 'force', 'compound', 'phlogiston', 'planets', 'mass', 'element', etc.) that have a greater level of generality than other kind-terms (such as 'alloy', 'metal', 'physical body', 'salts', 'gold', 'water') and have a central theoretical role in that they enter into the formulation of basic theoretical laws.
} 
a process not unlike speciation and one that I will later suggest is the reason for the ever-increasing specialization of the sciences' (Kuhn, 1993, p. 319).

Following from the above reading of Kuhn's insight, a primary type of unmatchable relationships between two taxonomic structures can be specified as follows:

Two taxonomic structures are unmatchable if the extensions of some shared theoretical kind-terms in two taxonomies overlap (but are not co-extensive) in some local area to some extent so that incorporating one into the other will directly violate the no-overlap principle. ${ }^{34}$

In addition to the above primary type of unmatchable relationships between two taxonomies, there exists at least another type: two taxonomic structures are mismatched to such an extent that they are either totally disjointed or lack any major overlap. Sometimes two competing languages may categorize a domain so differently that there is virtually no major overlapping between their taxonomies. B. Whorf has shown how different languages might categorize the world around themselves in totally different ways. Chinese medical theory and Western medical theory belong to such cases. It is hard to locate any major overlap between them since they employ two totally disjointed category systems at the theoretical level. Also, it is perfectly imaginable that two alien cultures may have two disjointed taxonomies, although it is very unlikely that this will happen frequently.

\section{Truth-value gaps and incommensurability}

It is time to put all the threads together for an overall picture of Kuhn's taxonomic interpretation of incommensurability.

(1) Human categorization is determined by different contextual factors, such as cultural, historical and linguistic factors, and varies widely across different contexts. The taxonomic structures of different scientific languages about the same subject matter can be totally different. The taxonomic structures of two successive rival scientific languages before or after a scientific revolution may change dramatically so that two structures become unmatchable. ${ }^{35}$

(2) Different taxonomic structures gain conceptual access to different sets of possible worlds consisting of different possible facts. The truth-value status of sentence

\footnotetext{
${ }^{34}$ Notice that the no-overlap prohibition only applies to the theoretical kind-terms with nomic expectations. The principle does not apply to the extensions of singular terms (names and definite descriptions) and must be weakened for the kind-terms with normic expectations, i.e., the generalizations that admit exceptions (only terms which belong to the same contrasting set are prohibited from overlapping in extensions). Therefore, the no-overlap prohibition is restricted within some local area of two overlapping taxonomies, and leaves most parts of them open for possible overlapping. That means that a major overlap between two unmatchable taxonomies is still possible.

35 According to Kuhn, the change of taxonomic structures due to the shift of the network of similarity relations is the hallmark of scientific revolutions. See Kuhn (1970a), pp. 92, 200; Kuhn (1970b), pp. 275276; Kuhn (1976), p. 195; Kuhn (1979), p. 416; Kuhn (1987), pp. 20-21.
} 
$P$ of one scientific language $\mathrm{L}_{1}$ is determined by whether $P$, when considered within another competing scientific language $\mathrm{L}_{2}$, describes a possible fact in a possible world conceptually accessible by $\mathrm{L}_{2}$. Therefore, truth-value conditions of sentences are taxonomic-structure-dependent.

(3) When the taxonomic structures of two competing scientific languages are unmatchable to one another, the two sets of possible worlds specified by them will be disjointed. Many possible facts within one set of possible worlds specified by one language would not count as possible facts when considered within the other language. Since the truth-value status of sentences is possible-fact-dependent, there would be a truth-value gap occurring between the two languages. This is exactly what has happened in the episodes of scientific revolutions. During these periods, scientific development turns out to depend on transitions to another disjointed set of possible worlds due to a switch to another unmatchable taxonomic structure. Is it, in these circumstances, appropriate to say that the members of the two communities live in different worlds? (Kuhn, 1988, pp. 13-15, 22-24).

(4) Accepting sentences in discourse as candidates for truth-or-falsity is an essential ingredient of any unproblematic linguistic communication. If there is a truthvalue gap between two languages, this minimal logical rule of any language game is violated. Thus the occurrence of a truth-value gap between two languages indicates that the communication between them is problematic and inevitably partial. 'The would-be communicants have encountered incommensurability' (Kuhn, 1993, p. 326).

This then is the argument for a particular reconstruction of Kuhn's new interpretation of incommensurability, which is a combination of a logical-semantic theory of taxonomy, a semantic theory of truth-value, and a truth-value-conditional theory of communication. According to this truth-value interpretation of incommensurability, two scientific languages are incommensurable when core sentences of one language, which have truth-values when considered within its own context, lack truth-values when considered within the context of the other due to their unmatchable taxonomic structures.

It might be argued that the above truth-value interpretation of Kuhn's thesis of incommensurability may turn out not to differ greatly from the translation-failure interpretation. The critique goes: well, perhaps your interpretation does grasp an important aspect of incommensurability, namely, the occurrence of a truth-value gap between two incommensurable languages. But truth-value gaps are exactly what incommensurable languages are supposed to lead to on the translation-failure interpretation. When there is a truth-value gap between two competing languages, truth-preserving translation between them inevitably fails; for there is no way to match up sentences with the same truth-values in two languages respectively. If so, the occurrence of truth-value gaps entails some species of untranslatability. Is the truth-value interpretation a real alternative to the translation-failure interpretation?

It should be admitted that the critic is right about one direction of the relationship between truth-value gaps and untranslatability. Truth-value gaps do entail some species of untranslatability (in the sense of truth-preserving translation). So incommensurable languages are necessarily untranslatable. This means that the truth-value 
interpretation can, as one will expect, do justice to the translation-failure interpretation, which should be considered as one of its advantages. However, the move from untranslatability to truth-value gaps is not guaranteed since truth-preserving is only necessary, but not sufficient, for mutual translation. In fact, it is not clear at all how meaning-reference variance between two languages can, on the translation-failure interpretation, lead to a truth-value gap. Clearly only change of the meanings of some constituents of a sentence does not make it truth-valueless. For instance, suppose that, if the contextual theory of meaning was right, the term 'mass' in a Newtonian sentence-for example, 'The mass of a particle does not change with the velocity of the particle'-has different meanings in Newtonian physics and relativity theory. If the sentence sounds truth-valueless when considered within the language of relativity theory, it is not because of some change of the meaning of the term, but because a universal principle presupposed by the sentence-namely, that properties such as shape, mass and period inhere in objects and change only by direct physical interactions-is suspended by the relativity theory. Therefore, untranslatability does not entail truth-value gaps. In addition, as has been argued elsewhere (Wang, 1998), untranslatability does not necessarily lead to a cross-language communication breakdown as a truth-value gap due to unmatchable taxonomic structures does. So untranslatability will not necessarily, and will not usually, lead to incommensurability. It can be concluded that the truth-value interpretation is not only distinct from the translation-failure interpretation. It also identifies the real source of incommensurability.

\section{Acknowledgements}

I am grateful for comments on an earlier draft of this paper by two anonymous referees.

\section{References}

Balzer, W. (1989). On incommensurability. In K. Gavroglu, Y. Goudaroulis, \& P. Nicolacopoulos (Eds.), Imre Lakatos and theories of scientific change (pp. 287-303). Dordrecht: Kluwer Academic Publishers.

Biagioli, M. (1990). The anthropology of incommensurability. Studies in History and Philosophy of Science, 21, 183-209.

Brown, H. (1983). Incommensurability. Inquiry, 26, 3-29.

Chen, X. (1997). Thomas Kuhn's latest notion of incommensurability. Journal for General Philosophy of Science, 28, 257-273.

Davidson, D. (1984). Inquiries into truth and interpretation. New York: Oxford University Press.

Doppelt, G. (1978). Kuhn's epistemological relativism: An interpretation and defense. Inquiry, 21, 33-86.

Fu, D. (1995). Higher taxonomy and higher incommensurability. Studies in History and Philosophy of Science, 26, 273-294.

Gaifman, H. (1975). Ontology and conceptual frameworks (part I). Erkenntnis, 9, 329-353.

Gaifman, H. (1976). Ontology and conceptual frameworks (part II). Erkenntnis, 10, 21-85.

Gaifman, H. (1984). Why language? In W. Balzer, D. A. Pearce, \& H. J. Schmidt (Eds.), Reduction in science: Structure, examples, philosophical problems (pp. 319-330). Boston: D. Reidel Publishing Company. 
Hacker, P. (1996). On Davidson's idea of a conceptual scheme. The Philosophical Quarterly, 46, $289-307$. Hacking, I. (1982). Language, truth and reason. In M. Hollis, \& S. Lukes (Eds.), Rationality and relativism (pp. 48-66). Boston: MIT Press.

Hacking, I. (1993). Working in a new world: The taxonomic solution. In P. Horwich (Ed.), World change: Thomas Kuhn and the nature of science (pp. 275-309). Boston: MIT Press.

Hoyningen-Huene, P. (1990). Kuhn's conception of incommensurability. Studies in History and Philosophy of Science, 21, 481-492.

Hoyningen-Huene, P. (1993). Reconstructing scientific revolutions (A. Levine, Trans.). Chicago: University of Chicago Press.

Horwich, P. (1990). Truth. Oxford: Basil Blackwell.

Hung, H. (1987). Incommensurability and inconsistency of language. Erkenntnis, 27, 323-352.

Kuhn, T. (1970a). The structure of scientific revolution (2nd ed.). Chicago: University of Chicago Press.

Kuhn, T. (1970b). Reflection on my critics. In I. Lakatos, \& A. Musgrave (Eds.), Criticism and growth of knowledge (pp. 231-278). Cambridge: Cambridge University Press.

Kuhn, T. (1976). Theory-change as structure-change: Comments on the Sneed formalism. Erkenntnis, 10, $179-199$.

Kuhn, T. (1977a). The essential tension. Chicago: University of Chicago Press.

Kuhn, T. (1977b). Second thoughts on paradigms. In F. Suppe (Ed.), The structure of scientific theories (2nd ed.) (pp. 459-517). Champaign: University of Illinois Press.

Kuhn, T. (1979). Metaphor in science. In A. Ortony (Ed.), Metaphor and thought (pp. 409-419). Cambridge: Cambridge University Press.

Kuhn, T. (1983). Commensurability, comparability, communicability. PSA 1982, 2, 669-688.

Kuhn, T. (1987). What are scientific revolutions? In L. Krüger, G. Gigerenzer, \& M. S. Morgan (Eds.), The probabilistic revolution Vol. 2: Ideas in the sciences (pp. 7-22). Boston: MIT Press.

Kuhn, T. (1988). Possible worlds in history of science. In S. Allen (Ed.), Possible worlds in humanities, arts and sciences (pp. 9-32). Berlin: Walter de Gruyter.

Kuhn, T. (1991). The road since structure. PSA 1990, 2, 3-13.

Kuhn, T. (1993). Afterwords. In P. Horwich (Ed.), World changes: Thomas Kuhn and the nature of science (pp. 311-341). Boston: MIT Press.

Malone, M. (1993). Incommensurability without relativism. Studies in History and Philosophy of Science, 24, 69-93.

Ramberg, B. (1989). Donald Davidson's philosophy of language. Oxford: Basil Blackwell.

Sankey, H. (1991). Incommensurability and the indeterminacy of translation. Australasian Journal of Philosophy, 69, 219-223.

Wang, X. (1998). A critique of the translational approach to incommensurability. Prima Philosophia, 11(3), 293-306.

Wang, X. (1999a). Is the notion of semantic presupposition empty? Dialogos, 73, 61-91.

Wang, X. (1999b). Truth-value gaps, ontological commitments, and incommensurability. Ann Arbor, MI: UMI Dissertation Services, A Bell \& Howell Company.

Williamson, T. (1994). Vagueness. New York: Routledge. 